\title{
Correction to: Stability of monoclinic phase in pure and Gd-doped HfO2: a hyperfine interaction study
}

\section{Debashis Banerjee ${ }^{1,2} \cdot$ Chandi Charan Dey ${ }^{3,4} \cdot$ Sk. Wasim Raja ${ }^{1,2} \cdot$ Ram Sewak $^{3,4}$. S. V. Thakare ${ }^{5}$. Raghunath Acharya ${ }^{4,6}$. Pradeep Kumar Pujari ${ }^{4,6}$}

Published online: 5 September 2019

(C) Springer Nature Switzerland AG 2019

\section{Correction to: Hyperfine Interactions (2019) 240:78 https://doi.org/10.1007/s10751-019-1614-7}

Due to technical constraints this article was published in volume 240:1 with erroneous article citation ID number 8 whereas this should have been $\mathbf{7 8}$ which is corrected as such. Springer Nature sincerely apologizes towards the author(s) for the inconvenience caused.

Publisher's note Springer Nature remains neutral with regard to jurisdictional claims in published maps and institutional affiliations

This article is part of the Topical Collection on Proceedings of the International Conference on Hyperfine Interactions and their Applications (HYPERFINE 2019), Goa, India, 10-15 February 2019 Edited by S. N. Mishra, P. L. Paulose and R. Palit

The online version of the original article can be found at https://doi.org/10.1007/s10751-019-1614-7

Debashis Banerjee

dbanerjee@vecc.gov.in

Chandi Charan Dey

chandicharan.dey@saha.ac.in

Sk. Wasim Raja

sw.raja@vecc.gov.in

Ram Sewak

ram.kashyap@saha.ac.in

S. V. Thakare

svt@barc.gov.in

Raghunath Acharya

racharya@barc.gov.in

Pradeep Kumar Pujari

pujari@barc.gov.in

Extended author information available on the last page of the article 


\section{Affiliations}

Debashis Banerjee ${ }^{1,2} \cdot$ Chandi Charan Dey ${ }^{3,4} \cdot$ Sk. Wasim Raja ${ }^{1,2} \cdot$ Ram Sewak $^{3,4}$. S. V. Thakare ${ }^{5}$. Raghunath Acharya ${ }^{4,6}$. Pradeep Kumar Pujari ${ }^{4,6}$

1 Radiochemistry Division (BARC), Variable Energy Cyclotron Centre, Kolkata 700064, India

2 Bhabha Atomic Research Centre, VECC, Kolkata 700064, India

3 Applied Nuclear Physics Division, Saha Institute of Nuclear Physics, Kolkata 700064, India

4 Homi Bhabha National Institute, Mumbai 400094, India

5 Radiopharmaceutical Division, Bhabha Atomic Research Centre, Mumbai 400085, India

6 Radiochemistry Division, Bhabha Atomic Research Centre, Mumbai 400085, India 